\title{
A QUASITOPOS CONTAINING CONV AND MET AS FULL SUBCATEGORIES
}

\author{
E. LOWEN \\ University of Brussels, V.U.B. \\ Departement Wiskunde \\ P1einlaan 2 \\ 1050 Brusse1s, BELGIUM \\ R. LOWEN \\ University of Antwerp, R.U.C.A. \\ Wiskundige Analyse \\ Groenenborgerlaan 171 \\ 2020 Antwerp, BELGIUM
}

(Received May 12, 1987 and in revised form November 12, 1987)

ABSTRACT. We show that convergence spaces with continuous maps and metric spaces with contractions, can be viewed as entities of the same kind. Both can be characterized by a "limit function" $\lambda$ which with each filter $\mathcal{F}$ associates a map $\lambda \mathcal{F}$ from the underlying set to the extended positive real line. Continuous maps and contractions can both be chal acterized as limit function preserving maps.

The properties common to both the convergence and metric case serve as a basis for tne definition of the category, CAP. We show that CAP is a quasitopos and that, apart from the categories CONV, of convergence spaces, and MET, of metric spaces, it also contains the category AP of approach spaces as nicely embedded subcategories.

KEY WORDS AND PHRASES. Limit, distance, convergence space, metric space, appruacn. space, cartesian closed, hereditary. CLASSIFICATION. 54B30, 54A20, 54E35.

1. INTRODUCTION.

In [17] the categories TOP of topological spaces and continuous maps and pq-MET ${ }^{n}$ of extended pseudo-quasi-metric spaces and non-expansive maps were embedded in a common supercategory. The idea behind this embedding being that topological spaces and metric spaces can be viewed as objects of the same type, in the sense that they both can be described by a "distance between points and sets". Starting with a pq-MET ${ }^{\infty}$ space (X,d) this distance is the usual one given by $\delta(x, A):=\inf _{d}(x, a)$. Starting with a topological space $(X, \mathcal{G})$ a distance can be defined by $\delta(\vec{x}, \bar{A}):=0$ if $x \in \bar{A}$ and $\delta(x, A):=\infty$ if $x \notin \bar{A}$. A notion of distance has been axiomatized in [17] in such a way as to generalize hoth the metric and topological cases and resulted in the definition of the category AP of approach spaces and contractions.

There are several advantages to this.

In the first place that of unification, e.g. the notions of compactness (in TOP)

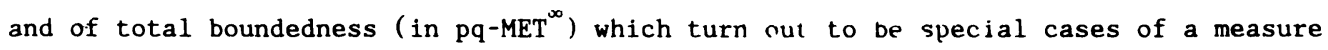


of compactness in AP [18] and which in turn makes a concept introduced by C. KURATOWSKI in [16] a canonical categorical notion.

A similar situation presents itself for the notions of connectedness (in TOP) and Cantor's "kettenzussamenhang" (in MET) [2], [19].

In the second place there are several classes of important topological spaces, e.g. spaces of measures with the weak topology and spaces of random variables with the topology of convergence in measure which can more naturally be equipped with AP-structures such that the topological structures are their TOP-coreflections [17].

In order to study these concepts and spaces it however soon became clear that we would need a theory of convergence in AP. We develop such a concept of convergence by means of assigning "limit functions" to filters, and moreover we show that AP can be completely characterized by four axioms about limit functions; two fundamental axioms -one on limit functions of principal ultrafilters and another on limit functions of comparable filters- a third axiom of a pretopological nature on limit functions of intersections of filters and a fourth one on limit functions of (Kowalsky-) diagonal filters [15].

Using this convergence-description of AP we obtain a very elegant characterization of initial structures in AP. AP is a topological construct in the sense of [1], [10], [11]. However from a categorical point of view some desirable properties are missing. For topologists and analysts, cartesian closedness is one such property [7], [9], [20], [21].

The existence of nice function space objects is indeed an important advantage in homotopy, topological algebra, and infinite dimensional differential calculus. The topological construct becomes extremely nice to work in when apart from being cartesian closed it also is hereditary, i.e. a quasitopos [5], [22], [23]. AP is neither cartesian closed nor hereditary. The situation is similar to the classical ones. Neither TOP nor pq-MET is a quasitopos. By weakening the axioms "bigger" categories with nicer properties result. For example CONV is a quasitopos containing TOP, and pqs-MET ${ }^{\infty}$ is a quasitopos containing $\mathrm{pq}-\mathrm{MET}^{\infty}$. By dropping the diagonal axiom and weakening the pretopological axiom on limit functions we introduce the supercategory CAP of convergence approach spaces. CAP is a quasitopos and moreover it contains both quasitopoi CONV and

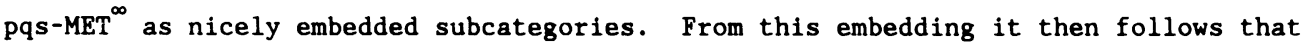
convergence spaces and extended pseudo-quasi-semi metric spaces can be viewed as entities of the same type, both being characterized by means of limit functions of filters. Moreover also AP is nicely embedded in this supercategory.

\section{PRELIMINARIES.}

If $X$ is a set then the set of all filters on $X$ shall be denoted $F(X)$. If $\mathcal{F} \in \mathbf{F}(X)$, then the set of all ultrafilters finer than $\mathcal{F}$ shall be $\operatorname{denoted} U(\mathcal{Z})$. If $\mathcal{Z}=\{X\}$ then we write shortly $\mathbf{U}(\mathrm{X})$.

For any collection $\alpha$ of subsets of $X$ we denote stack $a:=\{B \subset X \mid \exists A \in a$ : $A \subset B\}$. If $a$ consists of a single element $A$ we put shortly stack $A$ and if moreover $A$ consists of a single point a then we put stack $x^{a}$. If no confusion can occur, we drop the subscript and simply write stack $a$ a.s.o..

If $\left(s_{j}\right)_{j \in J}$ is a family of sets, then elements of their product $\Pi_{j \in J} s_{j}$ shall sometimes be denoted in a functional notation, e.g. s where for all $j \in J: s(j) \in S_{j}$. 
$\overline{\mathbb{R}}_{+}$stands for $[0, \infty]$ and all suprema and infima are taken in $\overline{\mathbb{R}}_{+}$. The following result shall be useful.

PROPOSITION 2.1. If $\mathcal{F} \in \mathbf{F}(\mathrm{X})$ and $\sigma \in \boldsymbol{\Pi} \boldsymbol{U}$ then there exists a finite set

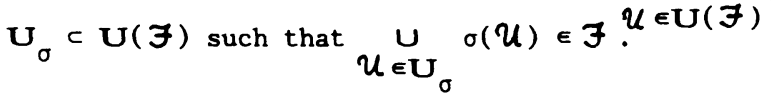

PROOF. Suppose not, in that case the family

$$
\mathcal{F} u\{X \backslash \sigma(\mathcal{U}) \mid \mathcal{U} \in \mathbf{U}(\boldsymbol{\xi})\}
$$

has the finite intersection property and thus is contained in some $\mathcal{U}_{0} \in U(\mathcal{J})$. Then however $\mathrm{X} \backslash \sigma\left(U_{0}\right) \in U_{0}$ which is a contradiction.

An extended pseudo-quasi-metric space (short1y o-pq-metric space) is a pair (X,d) where

$$
\mathrm{d}: \mathrm{X} \times \mathrm{X} \rightarrow \overline{\mathbb{R}}_{+}
$$

fulfils (i) $\forall x \in X: d(x, x)=0$ and (ii) $\forall x, y, z \in X: d(x, y) \leq d(x, z)+d(z, y)$. The map $d$ is called an extended pseudo-quasi-metric (shortly o-pq-metric). If $d$ is moreover symmetric, everywhere "quasi-" ("q-") is dropped.

Given two $\infty-p q-m e t r i c$ spaces $(X, d)$ and $\left(X^{\prime}, d^{\prime}\right)$ a function $f: X \rightarrow X^{\prime}$ is called non-expansive if $d^{\prime} \circ(f \times f) \leqq d$.

The category with objects $\infty$-pq-metric spaces and morphisms non-expansive maps is denoted pq-MET ${ }^{\infty}$. See also [13], [14].

An approach space is a pair $(X, \delta)$ where

$$
\delta: X \times 2^{\mathrm{X}} \rightarrow \overline{\mathbb{R}}_{+}
$$

fulfils

(D1) $\forall A \in 2^{X}, \forall x \in A: \delta(x, A)=0$

(D2) $\forall x \in X: \delta(x, \phi)=\infty$

(D3) $\forall \mathrm{A}, \mathrm{B} \in 2^{\mathrm{X}}, \forall \mathrm{x} \in \mathrm{X}: \delta(\mathrm{x}, \mathrm{A} \cup \mathrm{B})=\delta(\mathrm{x}, \mathrm{A}) \wedge \delta(\mathrm{x}, \mathrm{B})$

(D4) $\forall A \in 2^{X}, \forall x \in X, \forall \varepsilon \in \overline{\mathbb{R}}_{+}$:

$$
\delta(x, A) \leqq \delta\left(x, A^{(\varepsilon)}\right)+\varepsilon
$$

where $A^{(\varepsilon)}:=\{y \in X \mid \delta(y, A) \leqq \varepsilon\}$.

The map $\delta$ is called a distance.

Given $A \in 2^{X}$ we denote

$$
\begin{aligned}
\delta_{A} & : x \longrightarrow \overline{\mathbb{R}}_{+} \\
& : x \longmapsto \delta(x, A) .
\end{aligned}
$$

Given two approach spaces $(X, \delta)$ and $\left(X^{\prime}, \delta^{\prime}\right)$ a function $f: X \rightarrow X^{\prime}$ is called a contraction if for all $x \in X$ and $A \in 2^{X}$ :

$$
\delta^{\prime}(f(x), f(A)) \leq \delta(x, A)
$$

or equivalently, if for all $A \subset 2^{X}$ :

$$
\delta_{f(A)}^{\prime} \circ f \leqq \delta .
$$


3. CHARACTERIZATION OF AP VIA A CONVERGENCE THEORY.

In this section we shall give alternative characterizations of both approach spaces and contractions.

Let $\mathrm{X} \in|\mathrm{SET}|$. We recall the Kowalsky diagonal operator $D$ [15] defined as follows. For any index set $J$, any collection of filters $\left(\mathcal{F}_{j}\right)_{j \in J}$ on $x$, and filter $\mathcal{F}$ on $\mathrm{J}:$

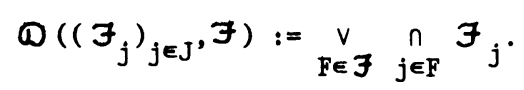

In the case the collection of filters is a selection on $X$ in the sense that we have a map

$$
\begin{aligned}
S: & x \rightarrow F(x) \\
& : x \rightarrow S(x)
\end{aligned}
$$

then we put shortly $\Phi(S, \exists)$ for $\Phi\left((S(y))_{y \in X}, \exists\right)$.

In the sequel we require the following results. Easy proofs are left to the reader.

PROPOSITION 3.1. Let $\left(\mathcal{F}_{j}\right)_{j \in J}$ be a collection of filters on $X$ and $\mathcal{F}$ a filter on $J$, then the following properties hold :

$1 \circ \emptyset\left(\left(\mathcal{F}_{j}\right)_{j \in J}, \mathcal{Z}\right)=\underset{F \in \mathcal{Z}}{U} \cap_{j \in F} \mathcal{Z}_{j}$.

$2^{\circ}$ If $\left(\mathcal{G}_{1}\right)_{1 \in \mathrm{L}}$ is a family of filters on $\mathrm{J}$ and $\mathcal{F}=\cap_{1 \in \mathrm{L}} \mathcal{S}_{1}$ then $\mathcal{Q}\left(\left(\mathcal{F}_{\mathrm{j}}\right)_{\mathrm{j} \in \mathrm{J}}, \mathcal{F}\right)=$ $\bigcap_{1 \in L} \omega\left(\left(\mathcal{F}_{j}\right)_{j \in J}, \mathcal{G}_{1}\right)$.

$\left.3^{\circ} \Phi\left(\mathcal{F}_{j}\right)_{j \in J}, \mathcal{F}\right)=\left(u_{j}\right)_{j \in J} \in \bigcap_{j \in J} U\left(\mathcal{F}_{j}\right)\left(\left(u_{j}\right)_{j \in J}, \mathcal{F}\right)$.

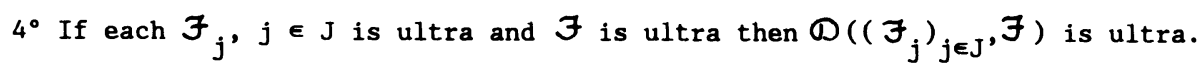

THEOREM 3.1. If $(X, \delta) \in|A P|$ then the map

$$
\begin{aligned}
\lambda: \mathbf{F}(\mathrm{X}) & \rightarrow \overline{\mathbb{R}}_{+}^{\mathrm{X}} \\
\mathcal{J} & \rightarrow \sup _{\mathcal{U \in U}(\mathcal{F}) \text { UE } \boldsymbol{U}} \delta_{U}
\end{aligned}
$$

fulfils

(CAL1) For any $x \in X: \lambda(\operatorname{stack} x)(x)=0$.

(CAL2) $\mathcal{F} \subset \mathcal{G} \Rightarrow \lambda(\mathcal{G}) \leqq \lambda(\mathcal{F})$.

(PRAL) For any family $\left(\mathcal{F}_{j}\right)_{j \in J}$ of filters on $X$ :

$$
\lambda\left(\operatorname{f\in J}_{j \in J}\right)=\sup _{j \in J} \lambda\left(\mathcal{F}_{j}\right) .
$$

(AL) For any $\mathcal{Z} \in \mathbf{F}(\mathrm{X})$ and any selection of filters $(S(y))_{y \in X}$ on $X$ :

$$
\lambda(の(S, \mathcal{F})) \leq \lambda(\mathcal{F})+\sup _{y \in X} \lambda(S(y))(y) .
$$

Moreover, for any $\mathrm{x} \in \mathrm{X}$ and $\mathrm{A} \subset \mathrm{X}$ :

$$
\delta(\mathrm{x}, \mathrm{A})=\underset{u \in U(\operatorname{stack} \mathrm{A})}{\inf } \lambda(\boldsymbol{u})(\mathrm{x}) .
$$

PROOF. (CAL1) follows from (D1) whereas (CAL2) follows from the fact that $\mathcal{F} \subset \mathcal{G}$ implies $\mathbf{U}(\mathcal{S}) \subset \mathbf{U}(\mathcal{F})$. 
To prove (PRAL), let $\left(\boldsymbol{\exists}_{j}\right)_{j \in J} \subset \mathbf{F}(X)$. One inequality follows from (CAL2), to show the other one observe that for all $u \in U\left(n_{j \in J} \mathcal{F}_{j}\right)$ and $U \in U$ there exists $j \in J$ and $\boldsymbol{W} \in U\left(\mathcal{F}_{j}\right)$ such that $U \in \mathcal{Y}$. Consequently we have

$$
\begin{aligned}
& \lambda\left(\bigcap_{j \in J} \mathcal{F}_{j}\right) \\
& =u \in U\left(\sum_{j \in J}^{\left.\sup _{j}\right)} \sup _{U \in U} \delta_{U}\right. \\
& =\sup _{j \in J} \sup _{\boldsymbol{U} \in\left(\xi_{j}\right)} \sup _{W \in W} \delta_{W} \\
& =\sup _{j \in J} \lambda\left(\mathcal{F}_{j}\right) \text {. }
\end{aligned}
$$

To prove (AL) let us first suppose that $\mathcal{Z} \in U(X)$ and that for all y $\in X$ :

$S(y) \in U(X)$ too. Now put $\varepsilon:=\sup _{y \in X} \lambda(S(y))(y)$. Then for any $D \in \Phi(S, 3)$, by Pro-

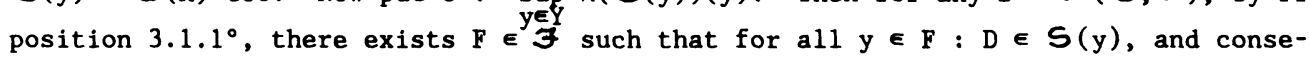
quent1y

$$
\delta(y, D) \leqq \lambda(S(y))(y) \leqq \varepsilon .
$$

Thus $D^{(\varepsilon)} \in \mathcal{F}$ and it follows from (D4) that

$$
\begin{aligned}
\delta_{D} & \leqq \delta_{D}(\varepsilon)+\varepsilon \\
& \leqq \sup _{F \in \mathcal{F}} \delta_{F}+\varepsilon \\
& \leqq \lambda(\exists)+\varepsilon .
\end{aligned}
$$

By the arbitrariness of $D \in D(S, \mathcal{B})$ and Proposition $3.1 .4^{\circ}$ it follows herefrom that

$$
\begin{aligned}
& \lambda \boldsymbol{Q}(\boldsymbol{S}, \boldsymbol{F})=\sup _{D \in \Phi(\boldsymbol{S}, \mathcal{F})}{ }^{\delta} \\
& \leq \lambda(\mathcal{F})+\varepsilon \text {. }
\end{aligned}
$$

Second, let us now suppose $\mathcal{F}$ and all $S(y), y \in X$ are arbitrary filters on $X$, let again $\varepsilon:=\sup _{y \in X} \lambda(S(y))(y)$ and for each $R \in \prod_{y \in X} U(S(y))$ let $\varepsilon_{R}:=\sup _{y \in X} \lambda(R(y))(y)$. Then by straightforward verification we have

$$
\varepsilon=\sup _{\mathrm{R} \in \mathrm{X}} \mathrm{U}(S(\mathrm{y}))^{\varepsilon_{R}} .
$$

By the foregoing result we know that for any $R \in \underset{y \in X}{ } U(S(y))$ and $u \in U(\mathcal{F})$ :

$$
\lambda(Q(R, u)) \leqq \lambda(u)+\varepsilon_{Q}
$$

Further, by Proposition $3.1 .2^{\circ}$ and $3^{\circ}$ we also have

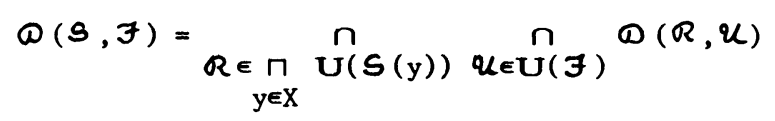

Combining (3.1), (3.2), (3.3) and upong applying (PRAL) it follows that

$$
\lambda(\square(\mathbf{S}, \mathcal{Z})) \leqq \lambda(\mathcal{Z})+\varepsilon \text {. }
$$

To prove the final claim of the theorem, first from the fact that for any 
$\mathcal{U} \in U($ stack $A)$ we have $A \in U$ one inequality is clear. Second, by definition of $\lambda$ and applying complete distributivity we obtain

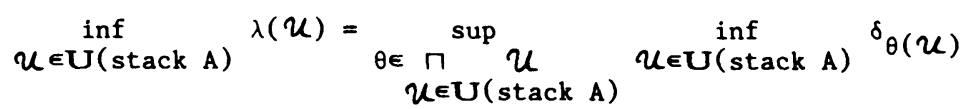

By Proposition 2.1, for each $\theta \in \underset{U \in U(\text { stack } A)}{U}$ we can find $U_{\theta} \subset \mathbf{U}$ (stack A) finite such that $A \subset \underset{U \in U_{\theta}}{U} \theta(U)$. Consequently by (D3), we obtain

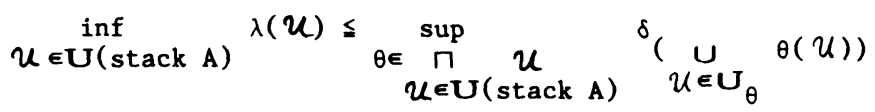

$$
\leqq \delta_{\mathrm{A}}
$$

which proves the remaining inequality.

THEOREM 3.2. If $X \in|S E T|$ and

$$
\lambda: \mathbf{F}(\mathrm{X}) \rightarrow \overline{\mathbb{R}}_{+}^{\mathrm{X}}
$$

is a map fulfilling (CAL1), (CAL2), (PRAL) and (AL) then the map

$$
\begin{aligned}
\delta: x \times 2^{X} & \rightarrow \overline{\mathbb{R}}_{+} \\
(x, A) & \rightarrow \inf _{U \in U(\operatorname{stack} A)} \lambda(U)(x)
\end{aligned}
$$

is a distance on $\mathrm{X}$.

Moreover, for any $\mathcal{F} \in \mathbf{F}(\mathrm{X})$ :

$$
\lambda(\mathcal{F})=\sup _{u \in U(\mathcal{J})} \sup _{U \in \mathcal{U}} \delta_{U} .
$$

PROOF. (D1) follows from (CAL1), (D2) follows from the fact that the infimum over an empty set is infinite, and (D3) follows from the fact that for any $A, B \subset X$ : $U($ stack $A \cup B)=U($ stack $A) \cup U($ stack $B)$. Before tackling (D4), we prove the final claim of the theorem. Let the map $\lambda^{\prime}$ be defined as

$$
\begin{aligned}
\lambda^{\prime}: \mathbf{F}(\mathrm{X}) & \rightarrow \overline{\mathbb{R}}_{+}^{\mathrm{X}} \\
\mathcal{F} & \longmapsto \sup _{U \in U(\mathcal{G})} \sup _{U \in \mathcal{U}} \delta_{U} .
\end{aligned}
$$

Now, let $u \in U(x)$. Then first we have

$$
\begin{aligned}
\lambda^{\prime}(u) & =\sup _{U \in \mathcal{U} U} \delta_{U} \\
& =\sup _{U \in U} \inf _{\forall \in U(\text { stack } U)} \lambda(\boldsymbol{W}) \\
& \leq \lambda(u) .
\end{aligned}
$$

Second, by complete distributivity and (PRAL) it follows that

$$
\begin{aligned}
\lambda^{\prime}(U) & =\sup _{U \in U} \inf \forall \in U(\text { stack } U) \\
& =\inf _{\theta \in \prod_{U \in U} U(\text { stack } U)} \lambda\left(\prod_{U \in U} \theta(U)\right) .
\end{aligned}
$$


Now for any $\theta \in \Pi \quad U($ stack $U)$ and any $U \in U$ we have $U \in \theta(U)$ and thus we have UE $u$

$n \theta(U) \subset u$ and it follows from (CAL2) that $\mathrm{U} \in \mathrm{U}$

$$
\lambda(u) \leq \lambda\left(\prod_{U \in U} \theta(U)\right) .
$$

By the arbitrariness of $\theta$ it follows in combination with (3.5) that

$$
\lambda(u) \leqq \lambda^{\prime}(u) \text {. }
$$

Together with (3.4) this shows that $\lambda$ and $\lambda^{\prime}$ coincide on ultrafilters.

By definition of $\lambda^{\prime}$ and the fact that $\lambda$ fulfils (PRAL) it then follows that $\lambda^{\prime}=\lambda$. In order to prove now (D4) let $A \subset X, \varepsilon \in \mathbb{R}_{+}$and choose any $\boldsymbol{W} \in U\left(A^{(\varepsilon)}\right)$. Now suppose that for some $y \in A^{(\varepsilon)}$ and for all $u \in U($ stack $A)$ :

$$
\begin{aligned}
\varepsilon & <\lambda(U)(y) \\
& =\lambda^{\prime}(U)(y)=\sup _{U \in \mathcal{U}} \delta(y, U) .
\end{aligned}
$$

This implies that for all $u \in U(\operatorname{stack} A)$ there exists $U_{u} \in U$ such that $\varepsilon<\delta\left(y, U_{u}\right)$. By Proposition 2.1 we can then find $u_{1}, \ldots, u_{n} \in U($ stack $A)$ such that $A \subset \bigcup_{i=1}^{n} u_{i}$ and then it follows from (D3) that

$$
\begin{aligned}
\varepsilon & <\inf _{i=1}^{n} \delta\left(y, U u_{1}\right) \\
& =\delta\left(y, \bigcup_{i=1}^{n} U_{u_{i}}\right) \\
& \leq \delta(y, A)
\end{aligned}
$$

which is in contradiction to the choice of $y$. Thus for all $y \in A^{(\varepsilon)}$ we can find $S(y) \in U($ stack A) such that

$$
\lambda(S(y))(y) \leqq \varepsilon .
$$

For $y \notin A(\varepsilon)$ put $S(y):=$ stack $y$ and then put

$$
\varepsilon^{\prime}:=\sup _{y \in X} \lambda(S(y))(y) \text {. }
$$

Now consider the diagonal filter $D(S, W)$ then $A \in \bigcap_{y \in A}(\varepsilon) S(y)$ and thus too

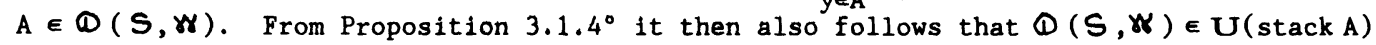
and from the definition of $\delta$ and (AL) it then follows further that for any $x \in X$ :

$$
\begin{aligned}
\delta(\mathbf{x}, A) & \leqq \lambda(\Phi(S, \boldsymbol{X}))(\mathbf{x}) \\
& \leqq \lambda(\boldsymbol{W})(\mathbf{x})+\varepsilon^{\prime} \\
& \leqq \lambda(\boldsymbol{W})(\mathbf{x})+\varepsilon .
\end{aligned}
$$

From the arbitrariness of $\delta \forall \in U\left(\operatorname{stack} A^{(\varepsilon)}\right.$ ) and the definition of $\delta$ it then finally follows that

$$
\delta(x, A) \leq \delta\left(x, A^{(\varepsilon)}\right)+\varepsilon .
$$

The combined results of Theorems 3.1 and 3.2 give yet another way to describe the objects of the category AP.

In what follows objects of AP shall then often also be denoted $(X, \lambda)$ where $\lambda$ then is a map on $F(X)$ fulfilling (CAL1), (CAL2), (PRAL) and (AL). We shall characterize the morphisms of AP using this new description of objects. 
THEOREM 3.3. If $(X, \lambda),\left(X^{\prime}, \lambda^{\prime}\right) \in|A P|$ and $f: X \rightarrow X^{\prime}$ is a function then the following are equivalent :

$1^{\circ} \mathrm{f}$ is a contraction

$2^{\circ} \forall \mathcal{F} \in \mathbf{F}(\mathrm{X}): \lambda^{\prime}($ stack $\mathrm{f}(\mathcal{F}))$ of $\leqq \lambda(\mathcal{F})$

$3^{\circ} \forall \mathcal{F} \in U(X): \lambda^{\prime}(\operatorname{stack} f(\mathcal{F}))$ of $\leqq \lambda(\mathcal{F})$.

PROOF. $1^{\circ} \Rightarrow 2^{\circ}$ : from Theorem 3.1 we obtain for any $\xi \in \mathbf{F}(\mathrm{X})$ :

$$
\begin{aligned}
& \lambda^{\prime}(\text { stack } f(\mathcal{F})) \circ f \\
& \left.=\sup _{\forall \in U(\operatorname{stack}} f(F)\right) \sup _{W \in W} \delta_{W}^{\prime} \circ f \\
& \leq \sup _{u \in U(\xi)} \sup _{U \in \mathcal{U}} \delta_{f(U)}^{\prime} \circ f \\
& \leqq \sup _{u \in U(\mathcal{Z})} \sup _{U \in U} \delta_{U} \\
& =\lambda(\mathcal{F}) \text {. }
\end{aligned}
$$

$2^{\circ} \Rightarrow 3^{\circ}:$ clear.

$3^{\circ} \Rightarrow 1^{\circ}$ : from Theorem 3.2 we obtain for any $\mathrm{A} \subset \mathrm{X}$ :

$$
\begin{aligned}
& \delta_{f(A)}^{\prime} \circ f=\inf _{\forall \in \in U(\operatorname{stack} f(A))} \lambda^{\prime}(\boldsymbol{W}) \circ f \\
& =\inf _{u \in U(\operatorname{stack} A)} \lambda^{\prime}(\text { stack } f(u)) \circ f \\
& =\inf _{u \in U(\operatorname{stack} A)}^{\lambda(u)} \\
& =\delta_{\mathrm{A}} \text {. }
\end{aligned}
$$

4. THE QUASITOPOS CAP

DEFINITION 4.1. Given $X \in|\mathrm{SET}|$ a map

$$
\lambda: \mathbf{F}(\mathrm{X}) \rightarrow \overline{\mathbb{R}}_{+}^{\mathrm{X}}
$$

is called a convergence-approach limit if it fulfils (CAL1), (CAL2) and the following weakening of (PRAL) :

(CAL3) For all $\mathcal{F}, \mathcal{S} \in \mathbf{F}(\mathrm{X}): \lambda(\mathcal{F} \cap \mathcal{S})=\lambda(\mathcal{F}) \vee \lambda(\mathscr{G})$. The pair $(\mathrm{X}, \lambda)$ is called a convergence-approach space.

DEFINITION 4.2. Given convergence-approach spaces $(X, \lambda)$ and $\left(X^{\prime}, \lambda^{\prime}\right)$ a function $f: X \rightarrow X^{\prime}$ is called a contraction if it fulfils :

(C)

$$
\text { For all } \mathcal{F} \in \mathbf{F}(\mathrm{X}): \lambda^{\prime}(\operatorname{stack} \mathrm{f}(\boldsymbol{\exists})) \text { of } \leqq \lambda(\mathcal{F}) \text {. }
$$

In the sequel, a convergence-approach limit and a convergence-approach space will be denoted shortly a CAP-limit and a CAP-space respectively.

We recall that a category of structured sets which is fibre-small and has the property that all constant maps between objects are morphisms is called a construct [1], $[11]$. 
If we denote CAP the category with objects all CAP-spaces and morphisms all contractions, then we obtain the following result, the verification of which is quite trivial.

PROPOSITION 4.1. CAP is a construct.

A construct is called topological [11] if it is finally (or equivalently initially) complete.

THEOREM 4.1. CAP is a topological construct.

PROOF. In order to show that CAP is initially complete consider the source

$$
\left(x \stackrel{f_{j}}{\longrightarrow}\left(x_{j}, \lambda_{j}\right)\right)_{j \in J}
$$

where all items have their obvious meaning.

Let $\lambda$ be defined by

$$
\begin{aligned}
\lambda: \mathbf{F}(\mathrm{X}) & \rightarrow \overline{\mathbb{R}}_{+}^{\mathrm{X}} \\
\exists & \rightarrow \sup _{j \in J} \lambda_{j}\left(\text { stack } \mathbf{f}_{j}(\exists)\right) \circ \mathbf{f}_{j} .
\end{aligned}
$$

To show that $\lambda$ is a CAP-1imit on $X$ is quite simple. (CAL1) and (CAL2) are trivial and (CAL3) follows from the observation that for any $j \in J$ and any $\mathcal{J}, \mathcal{S} \in \mathbf{F}(\mathrm{X})$, we have stack $f_{j}(\mathcal{Z} \cap S)=\operatorname{stack} f_{j}(\mathcal{Z}) \cap f_{j}(\mathcal{G})$.

To show that $\lambda$ is initial, let $\left(X^{\prime}, \lambda^{\prime}\right) \in|C A P|$ and let $\mathbf{g}: X^{\prime} \rightarrow X$ be a function such that for all $j \in J: f_{j} \circ g$ is a contraction. Then for any $\mathcal{F} \in \mathbf{F}\left(X^{\prime}\right)$ we have

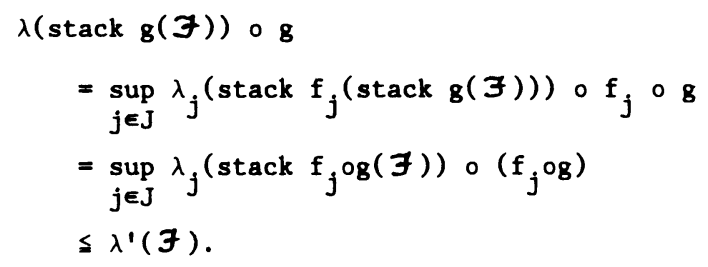

Consequently $g$ too is a contraction and we are done.

Before proceeding we now need some further notational conventions and definitions.

If $X, Y \in|C A P|$ then $\mathrm{HOM}_{\mathrm{CAP}}(\mathrm{X}, \mathrm{Y})$ stands for the set of all morphisms i.e. contractions from $X$ to $Y$. If no confusion can occur concerning the category under study we often omit the subscript and simply write $\operatorname{HOM}(X, Y)$.

Given $\Psi \in \mathbf{F}(\operatorname{HOM}(\mathrm{X}, \mathrm{Y}))$ and $\mathcal{F} \in \mathbf{F}(\mathrm{X})$ we define

$$
\Psi(\mathcal{F}):=\{\psi(F) \mid \psi \in \Psi, F \in \mathcal{F}\}
$$

where for all $\psi \in \Psi$ and $F \in \mathcal{F}$ :

$$
\psi(F):=\{g(y) \mid g \in \psi, y \in F\}
$$

Clearly, stack $\Psi(\mathcal{F}) \in \mathbf{F}(\mathrm{Y})$.

Next for any $f \in \operatorname{HOM}(X, Y)$ if $\lambda_{X}$ and $\lambda_{Y}$ are the CAP-limits on $X$ and $Y$ respectively, we defin.

$$
.(\vee, f):=\left\{\alpha \in \overline{\mathbb{R}}_{+} \mid \vee \mathcal{\exists} \in \mathbf{F}(\mathrm{X}): \lambda_{Y}(\operatorname{stack} \Psi(\mathcal{\exists})) \circ f \leq \lambda_{X}(\mathcal{F}) \vee \alpha\right\} \text {. }
$$


Quite obviously, $L(\Psi, f)$ is a subinterval of $\overline{\mathbb{R}}_{+}$and $\infty \in L(\Psi, f)$. Consequently the map

$$
\begin{aligned}
\lambda: \mathbf{F}(\operatorname{HOM}(\mathrm{X}, \mathrm{Y})) & \longrightarrow \overline{\mathbb{R}}_{+}^{\mathrm{HOM}(\mathrm{X}, \mathrm{Y})} \\
\Psi \longrightarrow \inf \mathrm{L}(\Psi, \cdot) & \longrightarrow
\end{aligned}
$$

is we11-defined.

PROPOSITION 4.2. $\lambda$ is a CAP-limit on $\operatorname{HOM}(\mathrm{X}, \mathrm{Y})$.

PROOF. We leave the details to the reader. (CAL1) follows from the fact that for any $f \in \operatorname{HOM}(X, Y)$ and any $\mathcal{F} \in \mathbf{F}(X): \operatorname{stack}(($ stack $f) \mathcal{F})=\operatorname{stack} f(\mathcal{F})$. (CAL2) and (CAL3) follow from the facts that for any $f \in \operatorname{HOM}(X, Y)$ and any $\Psi, \Phi \in F(H O M(X, Y))$ respectively, if $\Psi \subset \Phi$ then $L(\Psi, f) \subset L(\Phi, f)$ and if $\Psi$ and $\Phi$ are arbitrary then $L(\Psi \cap \Phi, f)=$ $L(\Psi, f) \cap L(\Phi, f)$.

If $G$ is a topological construct, then $G$ is called cartesian closed if for all objects $A, B \in|G|$, the set HOM $_{G}(A, B)$ can be endowed with a $G$-structure such that the evaluation map

$$
\text { ev : } \mathrm{A} \times \mathrm{HOM}_{\mathrm{G}}(\mathrm{A}, \mathrm{B}) \rightarrow \mathrm{B}
$$

defined by $\operatorname{ev}(a, f):=f(a)$ is co-universal with respect to the endofunctor $A \times-$. For more information on cartesian closedness, we refer to [7], [9], [20], [21] .

THEOREM 4.2. CAP is cartesian closed.

PROOF. The assertion we have to prove breaks up in two parts :

(1) For any two objects $\left(X, \lambda_{X}\right)$ and $\left(Y, \lambda_{Y}\right)$ in CAP and $\lambda$ as defined in Proposition 4.2, the evaluation

$$
\text { ev }:\left(X, \lambda_{X}\right) \times(\operatorname{HOM}(X, Y), \lambda) \rightarrow\left(X, \lambda_{Y}\right)
$$

is a contraction.

(2) For any three objects $\left(X, \lambda_{X}\right),\left(Y, \lambda_{Y}\right)$ and $\left(Z, \lambda_{Z}\right)$ in CAP and a contraction

$$
f:\left(X \times Z, \lambda_{X} \times \lambda_{Z}\right) \rightarrow\left(Y, \lambda_{Y}\right)
$$

the transpose

$$
\mathrm{f}^{*}:\left(\mathrm{Z}, \lambda_{\mathrm{Z}}\right) \rightarrow(\operatorname{HOM}(\mathrm{X}, \mathrm{Y}), \lambda)
$$

defined by $f^{*}(z)(x):=f(x, z)$ is a contraction.

In order to verify (1) let $\mathscr{W} \in \mathbf{F}(\mathrm{X} \times \mathrm{HOM}(\mathrm{X}, \mathrm{Y}))$ and put $\mathcal{Z}:=\operatorname{pr}_{1}(\mathcal{E})$ and $\Psi:=\operatorname{pr}_{2}(\mathbb{E})$ where

are the canonical projections.

$$
\mathrm{X} \times \operatorname{HOM}(\mathrm{X}, \mathrm{Y}) \underset{\mathrm{pr}_{2}}{\stackrel{\mathrm{pr}_{1}}{\longrightarrow}} \mathrm{XOM}(\mathrm{X}, \mathrm{Y})
$$

Now $f i x(x, f) \in X \times \operatorname{HOM}(X, Y)$ then from the definition of $\lambda$ and the construction of initial structures in CAP, it follows that

$$
\begin{gathered}
\left(\lambda_{X} \times \lambda\right)(\circlearrowleft)(x, f)=\lambda_{X}(\mathcal{F})(x) \vee \lambda(\Psi)(f) \\
=\inf \left\{\lambda_{X}(\mathcal{F})(x) \vee \alpha \mid \alpha \in L(\Psi, f)\right\} .
\end{gathered}
$$

From the definition of $L(\Psi, f)$ it follows that for any $\alpha \in L(\Psi, f)$ :

$$
\lambda_{Y}(\operatorname{stack} \Psi(\mathcal{J}))(f(x)) \leqq \lambda_{X}(\mathcal{F})(x) \vee \alpha .
$$


From (4.1), (4.2) and the fact that $\operatorname{ev}(\mathcal{3} \times \Psi)=\Psi(\mathcal{Z})$ it follows that

$$
\begin{aligned}
& \lambda_{Y}(\text { stack ev }(\mathcal{G}))(f(x)) \\
& \leqq \lambda_{Y}(\text { stack ev }(\mathcal{F} \times \Psi))(f(x)) \\
&=\lambda_{Y}(\text { stack } \Psi(\mathcal{J}))(f(x)) \\
& \leqq \lambda_{X}(\mathcal{F})(x) \vee \lambda(\Psi)(f) \\
&=\left(\lambda_{X} \times \lambda\right)(\mathcal{E})(x, f)
\end{aligned}
$$

This proves (1).

In order to verify (2) notice that for any $\mathcal{G} \in \mathbf{F}(\mathrm{Z}), \mathcal{F} \in \mathbf{F}(\mathrm{X}), \mathrm{z} \in \mathrm{Z}$ and $\mathrm{x} \in \mathrm{X}$, since $f \in \operatorname{HOM}(\mathrm{X} \times \mathrm{Z}, \mathrm{Y})$, we have

$$
\begin{aligned}
& \lambda_{Y}\left(\operatorname{stack} f^{*}(\mathcal{G})(\mathcal{\exists})\right)\left(f^{*}(z)(x)\right) \\
& \quad=\lambda_{Y}(\text { stack } f(\exists \times G))(f(x, z)) \\
& \leq \lambda_{X}(\exists)(x) \vee \lambda_{Z}(G)(z) .
\end{aligned}
$$

Consequently $\lambda_{Z}(G)(z) \in L\left(f^{*}(S), f^{*}(z)\right)$ which implies that

$$
\lambda\left(f^{*}(G)\right)\left(f^{*}(z)\right) \leqq \lambda_{Z}(G)(z) \text {. }
$$

The arbitrariness of $G$ and $z$ shows that $f^{*}$ is indeed again a contraction. This ends the proof of the theorem.

A topological construct is called hereditary provided final epi-sinks are hereditary, or equivalently as was shown in [11], if partial morphisms are representable. If $G$ is a construct and $A, B \in|G|$ then a partial morphism from $A$ to $B$ is a morphism $f \in \operatorname{HOM}_{G}(\mathrm{C}, \mathrm{B})$ where $\mathrm{C}$ is a subobject of $\mathrm{A}$.

If $G$ has subobjects then partial morphisms are representable if every object $B \in|G|$ can be embedded via the addition of a single point ${ }_{B}$ into an object $B^{\#} \in|G|$ such that for every partial morphism $f: C \rightarrow B$ from $A$ to $B$ the map

$$
\begin{array}{rl}
\mathrm{f}^{\#}: \mathrm{A} \longrightarrow \mathrm{B} & \\
\mathrm{a} & \mathrm{f}(\mathrm{a}) \quad \text { if } a \in \mathrm{C} \\
{ }_{\mathrm{B}} & \text { if } a \notin \mathrm{C}
\end{array}
$$

is a morphism in $G$. We shall use this characterization to prove our next result.

THEOREM 4.3. CAP is hereditáry.

PROOF. Let $\left(X, \lambda_{X}\right),(Y, \lambda) \in|C A P|$ and let $Z \subset X$. The subobject determined by $Z$ we shall denote $\left(Z, \lambda_{Z}\right)$ where then for any $\mathcal{\exists} \in \mathbf{F}(Z)$ :

$$
\lambda_{Z}(\mathcal{\exists})=\lambda_{X}\left(\operatorname{stack}_{X} \mathcal{F}\right)
$$

Let $f:\left(Z, \lambda_{Z}\right) \rightarrow(Y, \lambda)$ be a partial morphism from $(X, \lambda X)$ to $(Y, \lambda)$. Let $Y^{\#}:=Y \cup\left\{{ }^{\infty}\right\}$ where ${ }^{\infty} \mathrm{Y} \notin \mathrm{Y}$ and $\operatorname{def}$ ine

$$
\lambda^{\#}: \mathbf{F}\left(Y^{\#}\right) \rightarrow \overline{\mathbb{R}}_{+}^{\#}
$$

as follows. If $\mathcal{Z} \in \mathbf{F}\left(\mathrm{Y}^{\#}\right) \backslash\left\{\right.$ stack $\left._{\mathrm{Y}}^{\#}{ }^{\infty} \mathrm{Y}\right\}$ then 


$$
\lambda^{*}(\mathcal{J})(y):= \begin{cases}\lambda\left(\left.\mathcal{F}\right|_{Y}\right)(y) & \text { if } y \in Y \\ 0 & \text { if } y={ }_{Y}^{\infty}\end{cases}
$$

and

$$
\lambda^{*}\left(\text { stack }_{Y^{*}}{ }_{Y}\right)=0 .
$$

It is rather dreary but straightforward to verify that $\left(Y^{*}, \lambda^{*}\right) \in|\operatorname{CAP}|$ and that $(Y, \lambda)$ is embedded in $\left(Y^{*}, \lambda^{*}\right)$ by inclusion, so we omit this. Now we define

$$
\mathrm{f}^{*}:\left(\mathrm{X}, \lambda_{\mathrm{X}}\right) \rightarrow\left(\mathrm{Y}^{*}, \lambda^{*}\right)
$$

by $f^{*}(x)=f(x)$ if $x \in Z$ and $f^{*}(x)=\infty_{Y}$ if $x \in X \backslash Z$.

To show that $f^{\#}$ is a contraction, let $\mathcal{F} \in \mathbf{F}(X)$ and $x \in X$. If $\mathcal{F}$ has a trace on $Z$ then it is clear that stack $Y^{*} f^{*}(\mathcal{F})$ has a trace on $Y$ equal to stack $Y\left(\left.\mathcal{F}\right|_{Z}\right)$. If then $x \in Z$ it follows that $f^{*}(x)=f(x) \in Y$ and by definition of $\lambda^{*}$ and the fact that

$f \in \operatorname{HOM}(Z, Y)$ we then obtain

$$
\begin{aligned}
& \lambda^{*}\left(\text { stack }_{Y^{*}} f^{*}(\mathcal{J})\right)\left(f^{*}(x)\right) \\
& =\lambda\left(\text { stack }_{Y} f\left(\left.\mathcal{F}\right|_{Z}\right)\right)(f(x)) \\
& \leq \lambda_{Z}\left(\left.\mathcal{F}\right|_{Z}\right)(x) \\
& =\lambda_{X}\left(\operatorname{stack}_{X}(\mathcal{F} \mid Z)\right)(x) \\
& \leq \lambda_{X}(\mathcal{F})(x) .
\end{aligned}
$$

If $x \in X \backslash Z$ the same inequality results at once from the definition of $\lambda^{*}$ and from $f^{*}(x)=\infty_{Y}$. If $\mathcal{F}$ does not have a trace on $Z$ then again the same inequality holds for

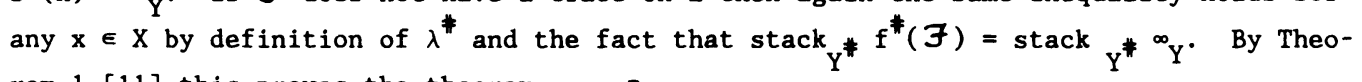
rem 1 [11] this proves the theorem.

Since by definition a quasitopos is a hereditary cartesian closed topological construct [10] our main result now is an immediate consequence of the foregoing theorems.

THEOREM 4.4. CAP is a quasitopos.

5. THE HEREDITARY TOPOLOGICAL CONSTRUCT PRAP

DEFINITION 5.1. Given $X \in \mid$ SET $\mid$ a map

$$
\lambda: \mathbf{F}(\mathrm{X}) \rightarrow \overline{\mathbb{R}}_{+}^{\mathrm{X}}
$$

is called a pre-approach limit (or PRAP-limit for short), if it fulfils (CAL1), (CAL2) and (PRAL). The pair $(X, \lambda)$ is then called a pre-approach space (or PRAP-space for short).

Clearly each pre-approach space is a convergence-approach space. The full subcategory of CAP with objects all pre-approach spaces shall be denoted PRAP. From Proposition 4.1 we at once obtain the next result.

PROPOSITION 5.1. PRAP is a construct.

In Theorems 3.1 and 3.2, we proved that giving a distance on a set $X$ is equivalent to giving an approach limit on $X$. A simple inspection of the proofs of these two theorems reveals that (D1), (D2) and (D3) are equivalent to (CAL1), (CAL2) and (PRAL). Consequently, if we call a map

$$
\delta: x \times 2^{\mathrm{X}} \rightarrow \overline{\mathbb{R}}_{+}
$$


fulfilling (D1), (D2) and (D3) a pre-distance, then without further proof we can state the following two results.

THEOREM 5.1. If $X \in|\mathrm{SET}|$ and $\delta$ is a pre-distance on $X$, then the map

$$
\begin{aligned}
\lambda: \mathbf{F}(\mathrm{X}) & \rightarrow \mathbb{\mathbb { R }}_{+}^{\mathrm{X}} \\
\mathcal{\exists} & \longmapsto \sup _{\mathcal{U} \in \mathbf{U}(\boldsymbol{\xi}) \sup _{U \in \mathcal{U}} \delta_{U}}
\end{aligned}
$$

is a pre-approach limit on $X$.

Moreover, for any $\mathrm{x} \in \mathrm{X}$ and $\mathrm{A} \subset \mathrm{X}$ :

$$
\delta(\mathrm{x}, \mathrm{A})=\inf _{u \in U(\operatorname{stack} A)} \lambda(u)(\mathrm{x}) .
$$

THEOREM 5.2. If $(X, \lambda) \in \mid$ PRAP $\mid$ then the map

$$
\begin{aligned}
\delta: & x \times 2^{\mathrm{X}} \rightarrow \overline{\mathbb{R}}_{+} \\
& (\mathrm{x}, \mathrm{A}) \rightarrow \inf _{u \in U(\operatorname{stack} A)} \lambda(u)(\mathrm{x})
\end{aligned}
$$

is a pre-distance on $X$.

Moreover, for any $\mathcal{F} \in \mathbf{F}(\mathrm{X})$ :

$$
\lambda(\mathcal{F})=\sup _{u \in \mathbf{U}(\mathcal{F})} \sup _{U \in \mathcal{U}} \delta_{U} .
$$

As was the case for approach spaces, the structure on a pre-approach space shall be determined either by a pre-approach limit or by a pre-distance, whichever is more convenient.

THEOREM 5.3. PRAP is a bireflective subcategory of CAP.

PROOF. Since PRAP contains all indiscrete CAP-objects, it will suffice to show that PRAP is initially closed in CAP. Let $\left(X_{j}, \lambda_{j}\right)_{j \in J}$ be a collection of PRAP-spaces and consider the source

$$
\left(x \stackrel{f_{j}}{\longrightarrow}\left(x_{j}, \lambda_{j}\right)\right)_{j \in J} \text {. }
$$

Let $\lambda$ be the initial CAP-limit on $X$ given by Theorem 4.1. To prove that $\lambda$ fulfils (PRAL), let $\left(\mathcal{F}_{k}\right)_{k \in K}$ be a collection of filters on $X$ then

$$
\begin{aligned}
\lambda\left(\bigcap_{k \in K} \mathcal{F}_{k}\right) & =\sup _{j \in J} \lambda_{j}\left(\operatorname{stack} f_{j}\left(\bigcap_{k \in K} \mathcal{F}_{k}\right)\right) \circ f_{j} \\
& =\sup _{j \in J} \lambda_{j}\left(\cap_{k \in K} \text { stack } f_{j}\left(\mathcal{F}_{k}\right)\right) \circ f_{j} \\
& =\sup _{j \in J} \sup _{k \in K} \lambda_{j}\left(\operatorname{stack} f_{j}\left(\mathcal{F}_{k}\right)\right) \circ f_{j} \\
& =\sup _{k \in K} \lambda\left(\mathcal{F}_{k}\right)
\end{aligned}
$$

and we are done.

REMARK. It is easily verified that the PRAP-reflection of a CAP-space $(X, \lambda)$ is given by

$$
(x, \lambda) \stackrel{i d_{x}}{\longrightarrow}\left(x, \lambda_{p}\right)
$$


where the pre-distance associated with $\lambda_{P}$ is given by

$$
\delta(\mathrm{x}, \mathrm{A}):=\inf _{u \in \mathrm{U}(\text { stack A })} \lambda(u)(\mathrm{x})
$$

COROLLARY 5.1. PRAP is a topological construct.

We shall later give a simple reason why PRAP is not cartesian closed, it is however hereditary as we shall now prove.

THEOREM 5.4. PRAP is an hereditary topological construct.

PROOF. The proof goes exactly the same as that of Theorem 4.3., the only difference being that now one starts with $\left(X, \lambda_{X}\right),(Y, \lambda) \in|P R A P|$ and one has to show that $\left(Y^{\#}, \lambda^{*}\right) \in|P R A P|$. We leave this to the reader.

6. EMBEDDING CONV IN CAP

A convergence space [6], [15] is a pair $(X, q)$ where $X \in|S E T|$ and $q \subset F(X) \times X$ fulfils

(C1) for all $x \in X:($ stack $x, x) \in q$.

(C2) For all $\mathcal{F}, \mathcal{G} \in \mathbf{F}(\mathrm{X})$ and $\mathrm{x} \in \mathrm{X}:(\mathcal{F}, \mathrm{x}) \in \mathrm{q}, \mathcal{F} \subset \mathcal{S} \Rightarrow(\mathcal{G}, \mathrm{x}) \in \mathrm{q}$.

(C3) For all $\mathcal{F}, \mathcal{S} \in \mathbf{F}(\mathrm{X})$ and $\mathrm{x} \in \mathrm{X}:(\mathcal{J}, \mathrm{x}) \in \mathrm{q}$ and $(\mathcal{G}, \mathrm{x}) \in \mathrm{q} \Rightarrow(\mathcal{F} \cap \mathcal{G}, \mathrm{x}) \in \mathrm{q}$.

Given convergence spaces $(X, q),\left(X^{\prime}, q^{\prime}\right)$ a function $f: X \rightarrow X^{\prime}$ is called continuous if for $\operatorname{all}(\mathcal{\exists}, \mathrm{x}) \in \mathrm{q}$ we have (stack $\mathrm{f}(\mathcal{\exists}), \mathrm{f}(\mathrm{x})) \in \mathrm{q}^{\prime}$.

The class with objects all convergence spaces and morphisms all continuous maps, is a quasitopos [10], denoted CONV.

The proof of the following result is quite straightforward and so we omit it. THEOREM 6.1. CONV is embedded as a full subcategory in CAP by the functor

$$
\begin{aligned}
& \mathrm{CONV} \rightarrow \mathrm{CAP} \\
& (\mathrm{X}, \mathrm{q}) \rightarrow\left(\mathrm{X}, \lambda_{\mathrm{q}}\right)
\end{aligned}
$$

where for all $\mathcal{Z} \in \mathbf{F}(\mathrm{X})$, and $\mathrm{x} \in \mathrm{X}$ :

$$
\lambda_{q}(\mathcal{F})(x):= \begin{cases}0 & \text { if }(\mathcal{F}, x) \in q \\ \infty & \text { otherwise. }\end{cases}
$$

We shall now show that this embedding actually is extremely nice, but first we mention the following useful characterization of CONV in CAP, similar to that of TOP in AP $[17]$.

PROPOSITION 6.1. A space $(\mathrm{X}, \lambda) \in|\mathrm{CAP}|$ is a convergence space, if and only if for all $\mathcal{F} \in \mathbf{F}(\mathrm{X}): \lambda(\mathcal{F})(\mathrm{X}) \subset\{0, \infty\}$.

As the formulation of this proposition suggests we shall not differentiate between the notion of a convergence space and of a CAP-space fulfilling the condition of Proposition 6.1. This is after all entirely justified by Theorem 6.1.

THEOREM 6.2. CONV is a bireflective subcategory of CAP.

PROOF. Given $(\mathrm{X}, \lambda) \in|\mathrm{CAP}|$ define

$$
\lambda_{*}: \mathbf{F}(\mathrm{X}) \rightarrow \overline{\mathbb{R}}_{+}^{\mathrm{X}}
$$


by $\lambda_{*}(\mathcal{F})(x)=0$ if $\lambda(\mathcal{F})(x)<\infty$ and $\lambda_{*}(\mathcal{F})(x)=\infty$ if $\lambda(\mathcal{F})(x)=\infty$. $\left(x, \lambda_{*}\right)$ clearly is a convergence space and the bireflection of $(X, \lambda)$ is given by

$$
(x, \lambda) \stackrel{i d_{X}}{\longrightarrow}\left(x, \lambda_{*}\right)
$$

THEOREM 6.3. CONV is a bicoreflective subcategory of CAP.

PROOF. Given $(X, \lambda) \in \mid$ CAP $\mid$ define

$$
\lambda^{*}: \mathbf{F}(\mathrm{X}) \rightarrow \overline{\mathbb{R}}_{+}^{\mathrm{X}}
$$

by $\lambda^{*}(\mathcal{F})(x)=0$ if $\lambda(\mathcal{F})(x)=0$ and $\lambda^{*}(\mathcal{F})(x)=\infty$ if $\lambda(\mathcal{F})(x)>0$. Again it is clear that $\left(X, \lambda^{*}\right)$ is a convergence space and that the bicoreflection of $(X, \lambda)$ is given by

$$
\left(x, \lambda^{*}\right) \stackrel{i d_{x}}{\longrightarrow}(x, \lambda)
$$

\section{EMBEDDING PRETOP IN PRAP}

A pre-topological space [4], [6] is a convergence space (X, q) where instead of (C3) q fulfils the stronger condition :

$$
\text { For any collection }\left(\mathcal{\exists}_{j}, x\right)_{j \in J} \subset q \text { we have }\left(\underset{j \in J}{n} \mathcal{F}_{j}, x\right) \in q \text {. }
$$

The full subcategory of CONV with objects all pre-topological spaces is denoted PRETOP. It is quite easy to see that precisely the same results hold for PRETOP w.r.t. PRAP, as those proven in Section 6 for CONV w.r.t. CAP. We therefore list them without further explanation.

THEOREM 7.1. PRETOP is embedded as a full subcategory in PRAP by the functor

$$
\begin{aligned}
& \text { PRETOP } \longrightarrow \text { PRAP } \\
& (\mathrm{x}, \mathrm{q}) \longmapsto\left(\mathrm{x}, \lambda_{\mathrm{q}}\right)
\end{aligned}
$$

where for all $\mathcal{F} \in \mathbf{F}(\mathrm{X})$ and $\mathrm{x} \in \mathrm{X}$ :

$$
\lambda_{q}(\exists)(x):= \begin{cases}0 & \text { if }(\exists, x) \in q \\ \infty & \text { otherwise. }\end{cases}
$$

PROPOSITION 7.1. A space $(\mathrm{X}, \lambda) \in|\mathrm{PRAP}|$ is a pre-topological space, if and only if for al1 $\mathcal{F} \in \mathbf{F}(\mathrm{X}): \lambda(\mathcal{F})(\mathrm{X}) \subset\{0, \infty\}$, or equivalently, if $\delta$ is the pre-distance associated with $\lambda$, if and only if $\delta\left(X \times 2^{X}\right) \subset\{0, \infty\}$.

THEOREM 7.2. PRETOP is a bireflective subcategory of PRAP, the bireflection of any PRAP-space being the same as its CONV-bireflection.

THEOREM 7.3. PRETOP is a bicoreflective subcategory of PRAP, the bicoreflection of any PRAP-space being the same as its CONV-bicoreflection.

Again, we shall not differentiate between pre-topological spaces and PRAP-spaces fulfilling the condition of Proposition 7.1. 
8. EMBEDDING AP IN PRAP

From Section 3 it is quite clear that AP is embedded as a full subcategory of PRAP.

THEOREM 8.1. AP is a bireflective subcategory of CAP.

PROOF. Since AP contains all indiscrete CAP-objects, it will suffice to show that AP is initially closed in CAP. Let $\left(X_{j}, \lambda_{j}\right){ }_{j \in J}$ be a family of AP-spaces and consider the source

$$
\left(x \stackrel{f_{j}}{\longrightarrow}\left(x_{j}, \lambda_{j}\right)\right)_{j \in J} \text {. }
$$

Let $\lambda$ be the initial CAP-1imit on X. From Theorem 5.3 we already know that $\lambda$ fulfils (PRAL). To show that it also fulfils (AL), let $\exists \in \mathbf{F}(X)$, let $(S(y))_{y \in X}$ be a selection of filters on $X$ an put $\varepsilon:=\sup \lambda(S(y))(y)$. Now, for all $j \in J$ define the following selection of filters on $x_{j} \stackrel{y \in X}{:}$

$$
R_{j}(z):= \begin{cases}\operatorname{y\in f}_{j}^{-1}(z) & \text { if } z \in f_{j}(x) \\ \text { stack } z & \text { if } z \notin f_{j}(x)\end{cases}
$$

We leave to the reader the straightforward verification that for all $j \in J$ :

$$
\Phi\left(\mathbb{R}_{j}, \text { stack } f_{j}(\mathcal{F})\right) \subset \text { stack } f_{j}(O(S, \mathcal{F})) \text {. }
$$

Next for all $j \in J$, put $\varepsilon_{j}:=\sup _{z \in X_{j}} \lambda_{j}\left(Q_{j}(z)\right)(z)$. Now if $z \notin f_{j}(X)$ then

$$
\lambda_{j}\left(R_{j}(z)\right)(z)=\lambda_{j}(\text { stack } z)(z)=0 \leqq \varepsilon
$$

whereas, if $z \in \mathrm{f}_{\mathrm{j}}(\mathrm{X})$ then

$$
\begin{aligned}
\lambda_{j}\left(R_{j}(z)\right)(z) & =\lambda_{j}\left(\underset{y \in f_{j}^{-1}(z)}{n} \operatorname{stack}_{j}(S(y))\right)(z) \\
& =\sup _{y \in f_{j}^{-1}(z)} \lambda_{j}\left(\text { stack } f_{i}(S(y))\right)\left(f_{j}(y)\right) \\
& \leqq \sup _{y \in X} \sup _{j \in J} \lambda_{j}\left(\text { stack } f_{j}(S(y))\right)\left(f_{j}(y)\right) \\
& =\varepsilon .
\end{aligned}
$$

By the arbitrariness of $j \in J$, this implies that

$$
\sup _{j \in J} \varepsilon_{j} \leqq \varepsilon .
$$

From (6.1) and (6.2) we then obtain

$$
\begin{aligned}
\lambda(\circlearrowleft(S, \mathcal{F})) & =\sup _{j \in J} \lambda_{j}\left(\text { stack } f_{j}(Ф(S, \exists))\right) \circ f_{j} \\
& \leqq \sup _{j \in J} \lambda_{j}\left(\Phi\left(Q_{j}, \text { stack } f_{j}(\exists)\right)\right) \circ f_{j}
\end{aligned}
$$




$$
\begin{aligned}
& \leqq \sup _{j \in J} \lambda_{j}\left(\operatorname{stack} f_{j}(\mathcal{\exists})\right) \circ f_{j}+\sup _{j \in J} \varepsilon_{j} \\
& \leqq \lambda(\exists)+\varepsilon
\end{aligned}
$$

and we are done.

COROLLARY 8.1. AP is a bireflective subcategory of PRAP.

9. EMBEDDING pqs-MET ${ }^{\infty}$ IN PRAP

The most general kind of map measuring a distance between points of a set $X$ is an extended pseudo quasi-semimetric (short1y o-pqs-metric). An o-pqs-metric

$$
\mathrm{d}: \mathrm{X} \times \mathrm{X} \rightarrow \overline{\mathbb{R}}_{+}
$$

need only fulfil $d(x, x)=0$ for all $x \in X$. The pair $(X, d)$ then is called an $\underline{\infty}$-pgsmetric space. Given $\infty$-pqs-metric spaces $(X, d)$ and $\left(X^{\prime}, d^{\prime}\right)$ a function $f: X \rightarrow X^{\prime}$ is called non-expansive if $d^{\prime} \circ(\mathrm{f} \times \mathrm{f}) \leqq \mathrm{d}$.

Let $\mathrm{pqs}^{-\mathrm{MET}^{\infty}}$ stand for the category with objects all $\infty$-pqs-metric spaces and morphisms al1 non-expansive maps.

THEOREM 9.1. pqs $-M E T^{\infty}$ is embedded as a full subcategory in PRAP by the functor

$$
\begin{gathered}
\text { pqs-MET }{ }^{\infty} \rightarrow \text { CAP } \\
(X, d) \rightarrow\left(X, \lambda_{d}\right)
\end{gathered}
$$

where for all $\mathcal{F} \in \mathbf{F}(\mathrm{X})$ and $\mathrm{x} \in \mathrm{X}$ :

$$
\lambda_{d}(\mathcal{Z})(x):=\inf _{F \in \mathcal{Z}} \sup _{y \in F} d(x, y)
$$

PROOF. That $\lambda_{d}$ fulfils (CAL1) and (CAL2) is clear. That it also fulfils (PRAL) is seen as follows. Let $\left(\mathcal{F}_{j}\right)_{j \in J} \subset \mathbf{F}(\mathrm{X})$ then for any $x \in X$ we have

$$
\begin{aligned}
\lambda_{d}\left(\prod_{j \in J} \mathcal{F}_{j}\right)(x) & =\inf _{F \in \prod_{j \in J} \mathcal{F}_{j} \sup _{y \in F} d(x, y)} \\
& =\inf _{\theta \in \prod_{j \in J} \mathcal{F}_{j} \sup _{j \in J} \theta(j)} d(x, y) \\
& =\inf _{\theta \in \prod_{j \in J} \mathcal{F u p}_{j} \sup _{j \in J} d(x, y)} d(j) \\
& =\sup _{j \in J} \lambda_{d}\left(\mathcal{F}_{j}\right)(x) .
\end{aligned}
$$

If $(X, d),\left(X^{\prime}, d^{\prime}\right) \in \mid$ pqs-MET ${ }^{\infty} \mid$ and $f:(X, d) \rightarrow\left(X^{\prime}, d^{\prime}\right)$ is non-expansive it is easily verified that $\mathrm{f}:\left(\mathrm{X}, \lambda_{\mathrm{d}}\right) \rightarrow\left(\mathrm{X}^{\prime}, \lambda_{\mathrm{d}^{\prime}}\right)$ is a contraction. The converse is equally simple upon noticing that from the definition of $\lambda_{d}$, for any $x, y \in X: d(x, y)=\lambda_{d}(\operatorname{stack} y)(x)$.

REMARK. By Theorems 5.1 and 5.2 , the pre-approach space $\left(X, \lambda_{d}\right)$ is identical to $\left(X, \delta_{d}\right)$ where $\delta_{d}$ is the pre-distance derived from $\lambda_{d}$, i.e. for all $x \in X$ and $A \subset X$ :

$$
\delta_{d}(x, A)=\inf _{u \in U(\operatorname{stack} A)} \inf _{U \in U} \sup _{y \in U} d(x, y) .
$$


This rather complicated expression for $\delta_{d}$ can however be much simplified using the following lemma.

LEMMA 9.1. Given $(X, d) \in\left|p q s-M E T^{\infty}\right|, u \in U(X)$ and $x \in X$ we have

$$
\sup _{U \in \mathcal{U}} \inf _{y \in U} d(x, y)=\inf _{U \in \mathcal{U}} \sup _{y \in U} d(x, y)
$$

PROOF. The inequality $\leqq$ follows from the fact that for any $U_{1}, U_{2} \in U$ :

inf $d(x, y) \leqq$ sup $d(x, y)$. To show the other one, suppose

$y \in U_{1} \quad y \in U_{2}$

$$
\inf _{U \in U} \sup _{y \in U} d(x, y)>\alpha \geq 0 .
$$

Then for all $U \in U$ there exists $y_{U} \in U: d\left(x, y_{U}\right)>\alpha$. Clearly

$$
\mathrm{w}:=\left\{\mathrm{y}_{U} \mid U \in U\right\} \in \mathcal{U}
$$

and $\underset{y \in W}{\inf } d(x, y) \geq \alpha$ which proves our claim.

THEOREM 9.2. Given $(\mathrm{X}, \mathrm{d}) \in\left|\mathrm{pqs}-\mathrm{MET}^{\infty}\right|$ the pre-distance $\delta_{\mathrm{d}}$ associated with $\lambda_{\mathrm{d}}$ is $\mathrm{g}$ iven by

$$
\delta_{d}(x, A)=\inf _{a \in A} d(x, a) \quad x \in X, A \subset X
$$

PROOF. Immediate from (9.1) and Lemma 9.1.

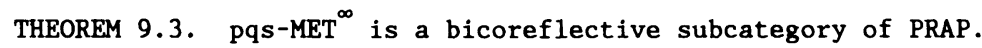

PROOF. Let $(X, \delta) \in \mid$ PRAP| and define the map

$$
\begin{aligned}
d_{\delta}: x \times x & \longrightarrow \overline{\mathbb{R}}_{+} \\
(x, y) & \longmapsto \delta(x,\{y\}) .
\end{aligned}
$$

It is clear that $\left(X, d_{\delta}\right) \in \mid$ pqs $-M^{\infty} T^{\infty} \mid$. The remainder of the proof now is exactly the same as in Theorem 6.7 [17], where it was shown that $\mathrm{pq}^{-\mathrm{MET}^{\infty}}$ is a bicoreflective subcategory of AP, and so we omit this.

Analogous to the characterization of $\mathrm{pq}-M E T^{\infty}$ in $\mathrm{AP}$ [17], we have the next result, the verification of which we leave to the reader.

PROPOSITION 9.1. A space $(X, \delta) \in|P R A P|$ is an $\infty$-pqs-metric space, if and only if for any $\mathrm{x} \in \mathrm{X}$ and $\mathrm{A} \subset \mathrm{X}$ :

$$
\delta(x, A)=\inf _{a \in A} \delta(x,\{a\}) .
$$

THEOREM 9.4. pqs-MET $^{\infty}$ is a hereditary topological construct.

PROOF. This is an immediate consequence of Corollary 5.1, Theorem 9.3 and of Theorem 6 $[11]$.

REMARK. Initial structures in $\mathrm{pqs}^{-\mathrm{MET}^{\infty}}$ are obtained as follows. Let

$$
\left(x \stackrel{f_{j}}{\longrightarrow}\left(x_{j}, d_{j}\right)\right)_{j \in J}
$$

be a source, then the initial $\infty$-pqs-metric on $\mathrm{X}$ is given by 


$$
d:=\sup _{j \in J} d_{j} \circ\left(f_{j} \times f_{j}\right) \text {. }
$$

We leave the verification to the reader.

THEOREM 9.5. Pqs-MET ${ }^{\infty}$ is cartesian closed.

PROOF. For $\left(X, d_{X}\right),\left(Y, d_{Y}\right) \in \mid$ pqs-MET ${ }^{\infty} \mid$ let HOM $(X, Y)$ stand for all non-expansive maps from $\left(X, d_{X}\right)$ to $\left(Y, d_{Y}\right)$. For any $f, g$ e $\operatorname{HOM}(X, Y)$ put

$$
C(f, g):=\left\{\alpha \in \overline{\mathbb{R}}_{+} \mid d_{Y} \circ(f \times g) \leqq d_{X} \vee \alpha\right\}
$$

and define

$$
\mathrm{d}: \operatorname{HOM}(\mathrm{X}, \mathrm{Y}) \times \operatorname{HOM}(\mathrm{X}, \mathrm{Y}) \longrightarrow \overline{\mathbb{R}}_{+}
$$

$$
(f, g) \longrightarrow \inf C(f, g)
$$

Clearly $d$ is a well-defined o-pqs-metric on $\operatorname{HOM}(X, Y)$. In order to show that

$$
\begin{aligned}
& \text { ev : }\left(X, d_{X}\right) \times(\operatorname{HOM}(X, Y), d) \rightarrow\left(Y, d_{Y}\right) \\
& (x, f) \longmapsto f(x)
\end{aligned}
$$

is non-expansive, let $x, y \in X$ and $f, g \in \operatorname{HOM}(X, Y)$ then

$$
\begin{aligned}
d_{Y}(f(x), g(y)) & \leq \inf \left\{d_{X}(x, y) \vee \alpha \mid \alpha \in C(f, g)\right\} \\
& =d_{X}(x, y) \vee d(f, g) \\
& =d_{X} \times d((x, y),(f, g)) .
\end{aligned}
$$

Next, if $\left(Z, d_{Z}\right) \in\left|p q s-M E T^{\infty}\right|$, $f \in \operatorname{HOM}(X \times Z, Y)$ then consider the map

$$
\begin{gathered}
f^{*}:\left(Z, d_{Z}\right) \\
z \rightarrow(\operatorname{HOM}(X, Y), d) \\
z f^{*}(z)
\end{gathered}
$$

where $f^{*}(z)$ is defined by $f^{*}(z)(x) t=f(x, z)$.

In order to show $f^{*}$ is non-expansive, let $z, z^{\prime} \in Z$ then since $f \in H O M(X \times Z, Y)$ we obtain for a11 $\mathrm{x}, \mathrm{x}^{\prime} \in \mathrm{X}$ :

$$
\begin{aligned}
d_{Y} & \circ\left(f^{*}(z) \times f^{*}\left(z^{\prime}\right)\right)\left(x, x^{\prime}\right) \\
& =d_{Y}\left(f(x, z), f\left(x^{\prime}, z^{\prime}\right)\right) \\
& \leqq d_{X}\left(x, x^{\prime}\right) \vee d_{Z}\left(z, z^{\prime}\right) .
\end{aligned}
$$

Consequently $d_{Z}\left(z, z^{\prime}\right) \in C\left(f^{*}(z), f^{*}\left(z^{\prime}\right)\right)$ and $d\left(f^{*}(z), f^{*}\left(z^{\prime}\right)\right) \leqq d_{Z}\left(z, z^{\prime}\right)$.

The combined results of Theorem 9.4 and 9.5 now give us the following theorem.

THEOREM 9.6. pqs-MET $^{\infty}$ is a quasitopos.

As a final result in this section, we shall now show that pq-MET (which is a bicoreflective subcategory of $\mathrm{AP}[17]$ ) is a bireflective subcategory of pqs-MET ${ }^{\infty}$.

THEOREM 9.7. $\mathrm{pq}^{-\mathrm{MET}^{\infty}}$ is a bireflective subcategory of pqs-MET ${ }^{\infty}$.

PROOF. Given $(X, d) \in \mid$ pqs $-M^{\infty} T^{\infty} \mid$ define

$$
\hat{\mathrm{d}}: \mathrm{X} \times \mathrm{X} \rightarrow \overline{\mathbb{R}}_{+}
$$

as follows. For any $\mathrm{x}, \mathrm{y} \in \mathrm{X}$, put 


$$
S_{n}(x, y):=\left\{\left(x_{j}\right)_{j=0}^{n} \in X^{n} \mid x_{0}=x, x_{n}=y\right\}
$$

and then define

$$
\hat{d}(x, y)=\inf _{n \in \mathbb{N}_{0}} \text { inf }\left\{\sum_{j=1}^{n} d\left(x_{j-1}, x_{j}\right) \mid\left(x_{j}\right){ }_{j=0}^{n} \in S_{n}(x, y)\right\} .
$$

Verification of the facts that $\hat{\mathrm{d}}$ satisfies the triangle inequality and that

$$
(x, d) \stackrel{i d x}{\longrightarrow}(x, \hat{d})
$$

is the $\mathrm{pq}^{-M E T^{\infty}}{ }^{-}$-reflection of $(X, d)$, we leave to the reader.

\section{COUNTEREXAMPLES}

COUNTEREXAMPLE 1. We construct a finitely generated topological space, a coNV-quotient of which is not in PRETOP. Let $\left.X:=\bigcup_{n \geq 3}\right] n, n+1[$. Consider the partition of $X$ by splitting each interval $] n, n+1[$ in three subintervals $] n, n+\frac{1}{n}\left[,\left[n+\frac{1}{n}, n+1-\frac{1}{n}\right],\right] n+1-\frac{1}{n}, n+1[$ and let $\mathcal{C}$ be the associated partition topology on $x$. Since $X$ is then a co-product of indiscrete spaces it is finitely generated [9]. Let $Y:=] 0,1[$ and consider the map

$$
\begin{aligned}
\mathrm{f}: & \mathrm{X} \\
& \rightarrow \mathrm{x}
\end{aligned}
$$

The conV-quotient $q$ on $Y$ is characterized by stating that $(\mathcal{F}, y) \in q$, if and only if there exist $\mathrm{x}_{1}, \ldots, \mathrm{x}_{\mathrm{n}} \in \mathrm{f}^{-1}(\mathrm{y})$ such that

$$
\prod_{i=1}^{n} f\left(\mathcal{L}\left(x_{i}\right)\right) \subset \exists
$$

where for any $x \in X: \mathcal{N}(\mathrm{x})$ is the $\zeta$-neighborhoodfilter of $x$. Consequently for all $\mathrm{n} \leqq 3$ :

but

$$
\begin{aligned}
& \left(f\left(\mathcal{W}\left(n+\frac{1}{2}\right)\right), \frac{1}{2}\right) \in q \\
& \left(\prod_{n \geq 3} f\left(\mathcal{W}\left(n+\frac{1}{2}\right)\right), \frac{1}{2}\right) \notin q
\end{aligned}
$$

and thus $\mathrm{q}$ is not pre-topological.

COUNTEREXAMPLE 2. We construct a finite o-pq-metric space, the $\infty$-pq-metric of which attains only the values 0 and $\infty$ and a pqs-MET ${ }^{\infty}$-quotient of which is not in Pq-MET ${ }^{\infty}$. Let $X:=\{u, v, x, y\}$ and let $d$ be the $\infty$-pq-metric (i.e. $d$ is symmetric) defined by

$$
\begin{gathered}
d(u, y)=d(v, x)=0 \\
d(u, v)=d(v, y)=d(y, x)=d(x, u)=\infty .
\end{gathered}
$$

Let $\mathrm{Y}:=\{\mathrm{u}, \mathrm{v}, \mathrm{z}\}$ and define the map

$$
\mathrm{f}: \mathrm{X} \rightarrow \mathrm{Y}
$$

by $f(u)=u, f(v)=v, f(x)=f(y)=z$.

If e denotes the pqs $-\mathrm{MET}^{\infty}$-quotient on $Y$ then the e-distance between two points in $Y$ is equal to the $d$-distance between their fibres. Consequently

$$
\begin{aligned}
& e(v, z)=d(\{v\},\{x, y\})=d(v, x) \wedge d(v, y)=0 \\
& e(u, z)=d(\{u\},\{x, y\})=d(u, x) \wedge d(u, y)=0
\end{aligned}
$$


but since

$$
e(u, v)=d(\{u\},\{v\})=d(u, v)=\infty
$$

e does not fulfil the triangle inequality and thus is not an o-pq-metric.

From [17] and the foregoing sections we obtain the following diagram where $r$ (resp. c) means the smaller category is bireflective (resp. bicoreflective) in the larger one.

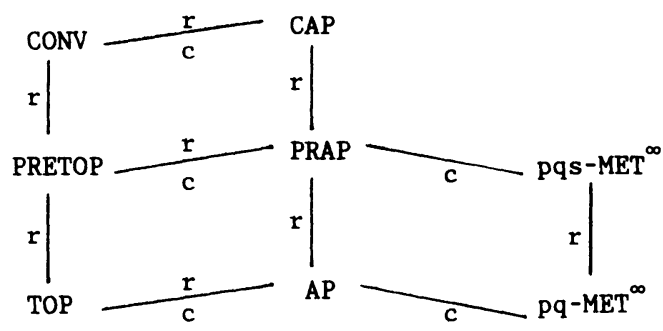

We shall now use the results of both counterexamples to show that this diagram is complete in the sense that no other reflectivity or coreflectivity arises except those indicated and those obtained by transitivity.

In [17] it was shown that TOP $\cap$ pq-MET ${ }^{\infty}$ consists precisely of all finitely generated spaces and that a distance attains at most the value 0 and $\infty$, if and only if it is associated with a topology. Consequently in both counterexamples the original space $X$ is at the same time in TOP, in $\mathrm{Pq}-\mathrm{MET}^{\infty}$ and a fortiori also in AP.

The first counterexample then shows that $Y$, the CONV-quotient (= CAP-quotient) of $X$, is not in PRETOP and since CONV $\cap$ PRAP = PRETOP (see Proposition 6.1 and 7.1) also not in PRAP. This gives us first the known results that neither TOP nor PRETOP are coreflective in CONV, second that neither AP nor PRAP are coreflective in CAP and third that neither $\mathrm{pq}-\mathrm{MET}^{\infty}$ nor $\mathrm{pqs}-\mathrm{MET}^{\infty}$ are coreflective in CAP.

The second counterexample gives us $Y$, the pqs-MET ${ }^{\infty}$-quotient (= PRAP-quotient) of $X$, which is not in $\mathrm{pq}^{-M E T}{ }^{\infty}$. However since the pre-distance on $Y$ again attains only the values 0 and $\infty, Y$ is in PRETOP. On the other hand, since pqs-MET $\cap A P=$ pq-MET $^{\infty}$ (see Theorem 6.20 [17] and Proposition 9.1) $Y$ is not in AP and thus also not in TOP. This then gives us first again the known result that TOP is not coreflective in PRETOP, second that $A P$ is not coreflective in $\mathrm{PRAP}$ and third that $\mathrm{Pq}^{-M E T}{ }^{\infty}$ is not coreflective in pqs-MET $^{\infty}$. Further in [17] it was seen that pq-MET is not reflective in AP from which it follows that pqs-MET ${ }^{\infty}$ is neither reflective in PRAP nor in CAP, and that $\mathrm{Pq}^{-M E T}{ }^{\infty}$ is not reflective in CAP. This completes our argumentation.

The authors would like to thank J. ADAMEK and L.D. NEL for bringing the problem of cartesian closedness in the setting of AP to their attention.

\section{REFERENCES}

1. ADAMEK J., Theory of mathematical structures, D. Reidel Publishing Company, 1983.

2. ARHANGELSKI A., WIEGANDT R., Connectedness and disconnectedness in topology, Gen. Top. App1. 5 (1975), 9-33.

3. BINZ E., Continuous convergence on C(X), Springer Lecture Notes in Math. 469 (1975).

4. CHOQUET G., Convergences, Ann. Univ. Grenoble 23 (1947/48), 57-112. 
5. DUBUC E.J., Concrete yuasitopoi, Springer Lecture Notes in Math. 753 (1979), 239254.

6. FISHER H.R., Limesraüme, Math. Ann. 137 (1959), 269-303.

7. HERRLICH H., Cartesian closed topological categories, Math. Collog. Univ. Cape Town 9 (1974), 1-16.

8. HERRLICH H., Are there convenient subcategories of TOP ? Top. App1. 15 (1983), 263-271.

9. HERRLICH H., Categorical topology 1971-1981, Proc. Fifth Prague Topol. Symp. 1981, Heldermann Verlag, Berlin (1983), 279-383.

10. HERRLICH H., Topological improvements of categories of structured sets, Topology App1. 27 (1987), 145-155.

11. HERRLICH H., Hereditary topological constructs, to appear.

12. HERRLICH H., G. STRECKER, Category theory, Allyn and Bacon (1973).

13. HOFFMANN R.E., Topological functors admitting generalized Cauchy-completions, Springer Lecture Notes in Math. 540 (1976), 286-344.

14. HOFFMANN R.E., Note in uiversal topological completion, Cahiers Top. Géom. 20 (1979), 216-229.

15. KOWALSKY H.J., Limesraüe und Komplettierung, Math. Nachr. 12 (1954), 301-340.

16. KURATOWSKI C., Sur les espaces complets, Fund. Math. 15 (1930), 301-309.

17. LOWEN R., Approach spaces : a common supercategory of TOP and MET, Math. Nachr., in print.

18. LOWEN R., Kuratowski's measure of non-compactness revisited, Quart. J. Math. Oxford, in print.

19. LOWEN R., Cantor's kettenzussamenhang revisited, to appear.

20. NEL L.D., Initially structured categories and cartesian closedness, Canad.J. Math. $\underline{27}(1975), 1361-1377$.

21. NEL L.D. , Cartesian closed topological categories, Springer Lecture Notes in Math.
540 (1976), 439-451.

22. NEL L.D. Topological universes and smooth Gelfand-Naimark duality, Contemporary
Math. 30 (1984), 244-276.

23. PENON J., Sur les quasi-topos, Cahiers Top. Géom. Diff. 18 (1977), 181-218. 


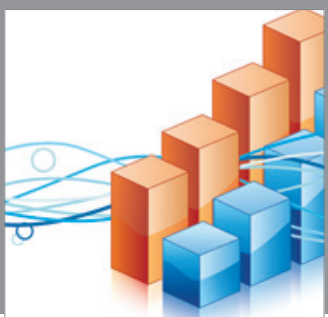

Advances in

Operations Research

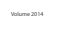

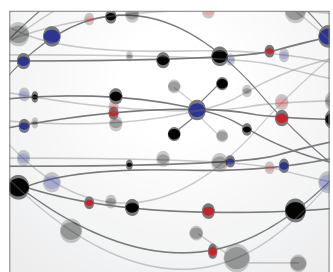

\section{The Scientific} World Journal
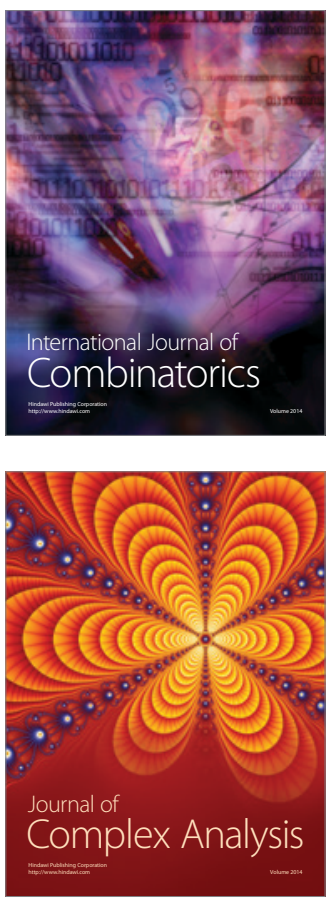

International Journal of

Mathematics and

Mathematical

Sciences
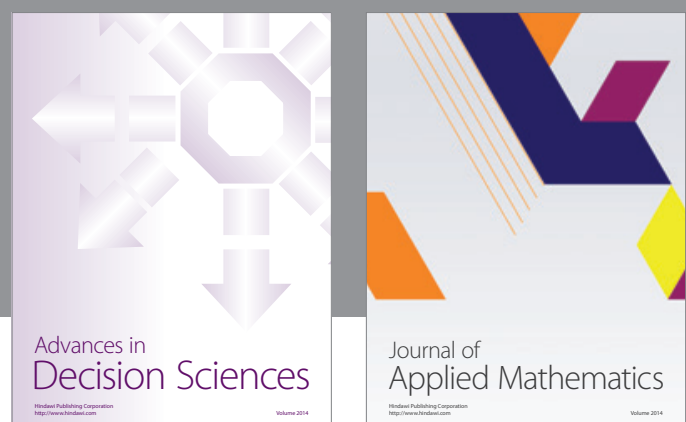

Journal of

Applied Mathematics
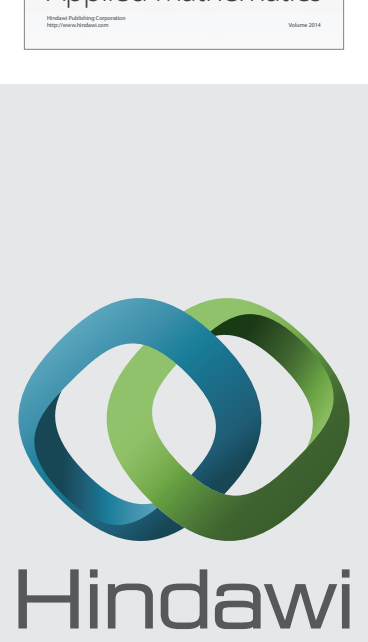

Submit your manuscripts at http://www.hindawi.com
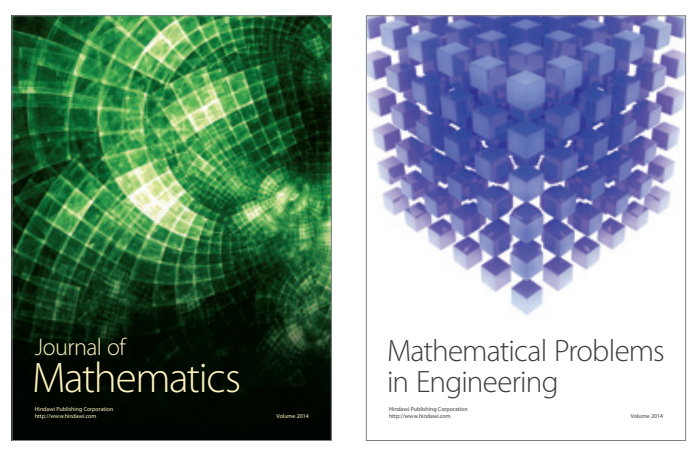

Mathematical Problems in Engineering
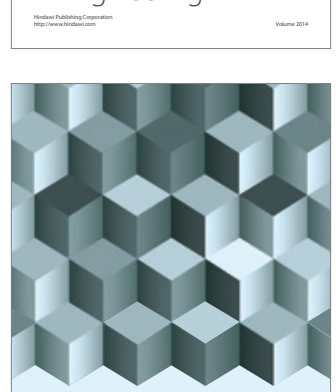

Journal of

Function Spaces
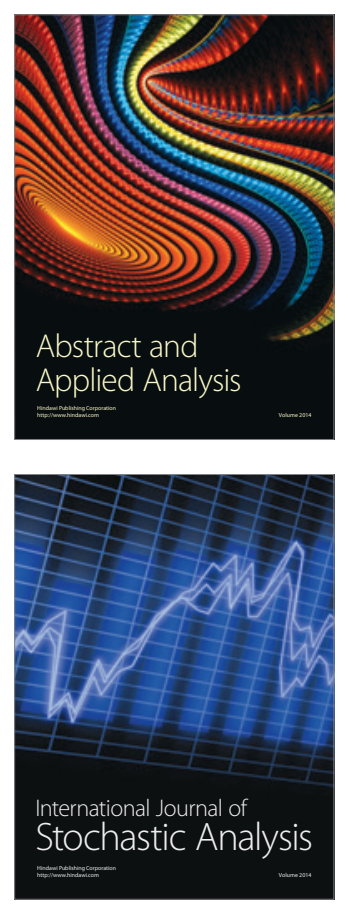

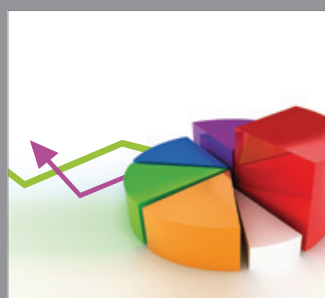

ournal of

Probability and Statistics

Promensencen
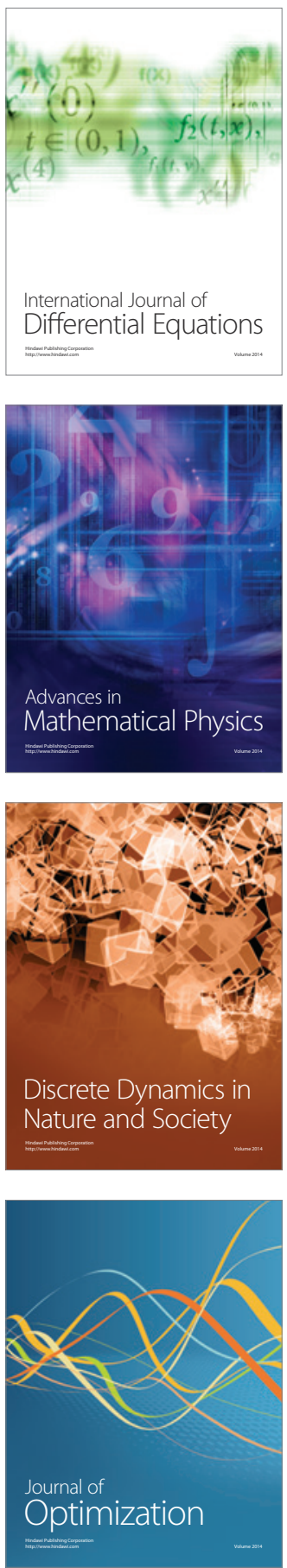